\title{
Pairing and nonaxial-shape correlations in $N=150$ isotones
}

\author{
Kenichi Yoshidd* \\ Department of Physics, Kyoto University, Kyoto, 606-8502, Japan
}

(Dated: May 10, 2021)

\begin{abstract}
Background: Rotational bands have been measured around ${ }^{250} \mathrm{Fm}$ associated with strong deformed-shell closures. The $K^{\pi}=2^{-}$excited band emerges systematically in $N=150$ isotones raging from plutonium to nobelium with even- $Z$ numbers, and a sharp drop in energies was observed in californium.

Purpose: I attempt to uncover the microscopic mechanism for the appearance of such a low-energy $2^{-}$state in ${ }^{248} \mathrm{Cf}$. Furthermore, I investigate the possible occurrence of the low-energy $K^{\pi}=2^{+}$state, the $\gamma$ vibration, to elucidate the mechanism that prefers the simultaneous breaking of the reflection and axial symmetry to the breaking of the axial symmetry alone in this mass region.
\end{abstract}

Method: I employ a nuclear energy-density functional (EDF) method: the Skyrme-Kohn-Sham-Bogoliubov and the quasiparticle random-phase approximation are used to describe the ground state and the transition to excited states.

Results: The Skyrme-type SkM* and SLy4 functionals reproduce the fall in energy, but not the absolute value, of the $K^{\pi}=2^{-}$state at $Z=98$, where the proton two-quasiparticle (2qp) excitation [633]7/2 $\otimes[521] 3 / 2$ plays a decisive role for the peculiar isotonic dependence. I find interweaving roles by the pairing correlation of protons and the deformed shell closure at $Z=98$. The SkM* model predicts the $K^{\pi}=2^{-}$state appears lower in energy in ${ }^{246} \mathrm{Cf}$ than in ${ }^{248} \mathrm{Cf}$ as the Fermi level of neutrons is located in between the [622]5/2 and [734]9/2 orbitals. Except for ${ }^{250} \mathrm{Fm}$ in the $\mathrm{SkM}^{*}$ calculation, the $K^{\pi}=2^{+}$state is predicted to appear higher in energy than the $K^{\pi}=2^{-}$state because the quasi-proton [521]1/2 orbital is located above the [633]7/2 orbital.

Conclusions: A systematic study of low-lying collective states in heavy actinide nuclei provides a rigorous testing ground for microscopic nuclear models. The present study shows a need for improvements in the EDFs to describe pairing correlations and shell structures in heavy nuclei, that are indispensable in predicting the heaviest nuclei.

\section{INTRODUCTION}

Recent developments in experimental technology and theoretical modeling have significantly advanced the study of exotic nuclei. The existence of superheavy nuclei in the limit of a great number of protons has long been a subject of interest in nuclear physics [1 3. The shell effect is a key to define their stability. The pairing correlations further play an influential role in determining the stability and shape of the ground state, and accordingly, the height of the fission barrier [4. However, it is not simple to investigate the shell structure and the pairing in such nuclei in the extreme regime. Strongly deformed actinide nuclei have thus been investigated as they can reveal the nuclear-structure information in the island of enhanced stability of spherical superheavy nuclei [5].

Details of the spectroscopic information in the actinide nuclei have provided an excellent testing ground for the reliability and predicting power of modern nuclearstructure theories [6, 7]. Several deformed shell closures are expected to emerge at $Z=98$ and 100 and at $N=150$ and 152 in the actinide nuclei 8]. Correspondingly, beautiful rotational bands have been measured [6, 7], that are indicative of an axially-deformed rotor. The rotational properties and the high- $K$ isomers have been studied in detail in terms of the pairing $99-13$.

In the course of the studies, anomalies have been revealed that are caused by residual interactions beyond

\footnotetext{
* E-mail: kyoshida@ruby.scphys.kyoto-u.ac.jp
}

the mean-field picture. A collective phenomenon shows up as a low-lying $K^{\pi}=2^{-}$state in the $N=150$ isotones, and the energy falls peculiarly in ${ }^{248} \mathrm{Cf}$ [14, 15]. The nonaxial reflection-asymmetric $\beta_{32}$ (dynamic) fluctuation and (static) deformation have been suggested by the projected shell-model calculation [16] and the relativistic energy-density-functional (EDF) calculation [17]. The quasiparticle-random-phase approximation (QRPA) calculations have also been performed to investigate the vibrational character [18, 19]. While the calculation using the Nilsson potential describes the anomalous behavior in ${ }^{248} \mathrm{Cf}$, the selfconsistent QRPA calculation employing the Gogny-D1M EDF shows a smooth isotonic dependence.

The observation of a strong $\beta_{32}$ correlation that breaks the axial symmetry suggests that the non-axial quadrupole deformation, $\gamma$ deformation, may also occur. Nuclear triaxiality brings about exotic collective modes: the appearance of the low-energy $K^{\pi}=2^{+}$band, the $\gamma$ band, is a good indicator for the $\gamma$ deformation [20, 21]. The $\gamma$ vibrational mode of excitation is regarded as a precursory soft mode of the permanent $\gamma$ deformation. Experimentally, the $K^{\pi}=2^{+}$state has not been observed to be as low in energy as the $K^{\pi}=2^{-}$state in ${ }^{244} \mathrm{Pu}$ and ${ }^{246} \mathrm{Cm} 22,23$. Therefore, it is interesting to investigate a microscopic mechanism that prefers the simultaneous breaking of the reflection and axial symmetry to the breaking of the axial symmetry alone.

Pairing correlations are essential for describing lowenergy vibrational modes of excitation 24] and are a key to understanding the collective nature. Therefore, in this 
article, I am going to investigate the role of the pairing correlations in both the static and dynamic aspects, as done in the studies in exotic nuclei in the neutron-rich region [25 27]. To this end, I employ a nuclear EDF method in which the pairing and deformation are simultaneously considered. Since the nuclear EDF method is a theoretical model being capable of handling nuclides with arbitrary mass numbers in a single framework [28, 29], the present investigation in the actinides can give an insight into the veiled nuclear structure of superheavy nuclei.

This paper is organized in the following way: the theoretical framework for describing the low-lying vibrations is given in Sec. II and details of the numerical calculation are also given; Sec. III is devoted to the numerical results and discussion based on the model calculation; after the discussion on the ground-state properties: the static aspects of pairing and deformation in Sec. III A. the discussion on the $K^{\pi}=2^{-}$and $K^{\pi}=2^{+}$states is given in Sec. III B 1 and Sec. III B 2 respectively; then, a summary is given in Sec. IV.

\section{THEORETICAL MODEL}

\section{A. KSB and QRPA calculations}

Since the details of the framework can be found in Refs. 30, 31, here I briefly recapitulate the basic equations relevant to the present study. In the framework of the nuclear EDF method I employ, the ground state is described by solving the Kohn-Sham-Bogoliubov (KSB) equation 32]:

$$
\begin{aligned}
& \sum_{\sigma^{\prime}}\left[\begin{array}{cc}
h_{\sigma \sigma^{\prime}}^{q}(\boldsymbol{r})-\lambda^{q} \delta_{\sigma \sigma^{\prime}} & \tilde{h}_{\sigma \sigma^{\prime}}^{q}(\boldsymbol{r}) \\
\tilde{h}_{\sigma \sigma^{\prime}}^{q}(\boldsymbol{r}) & -h_{\sigma \sigma^{\prime}}^{q}(\boldsymbol{r})+\lambda^{q} \delta_{\sigma \sigma^{\prime}}
\end{array}\right]\left[\begin{array}{c}
\varphi_{1, \alpha}^{q}\left(\boldsymbol{r} \sigma^{\prime}\right) \\
\varphi_{2, \alpha}^{q}\left(\boldsymbol{r} \sigma^{\prime}\right)
\end{array}\right] \\
& =E_{\alpha}\left[\begin{array}{c}
\varphi_{1, \alpha}^{q}(\boldsymbol{r} \sigma) \\
\varphi_{2, \alpha}^{q}(\boldsymbol{r} \sigma)
\end{array}\right]
\end{aligned}
$$

where the single-particle and pair Hamiltonians $h_{\sigma \sigma^{\prime}}^{q}(\boldsymbol{r})$ and $\tilde{h}_{\sigma \sigma^{\prime}}^{q}(\boldsymbol{r})$ are given by the functional derivative of the EDF with respect to the particle density and the pair density, respectively. An explicit expression of the Hamiltonians is found in the Appendix of Ref. [33]. The superscript $q$ denotes $\nu$ (neutron, $t_{z}=1 / 2$ ) or $\pi$ (proton, $\left.t_{z}=-1 / 2\right)$. The average particle number is fixed at the desired value by adjusting the chemical potential $\lambda^{q}$. Assuming the system is axially symmetric, the KSB equation (1) is block diagonalized according to the quantum number $\Omega$, the $z$-component of the angular momentum.

The excited states $|i\rangle$ are described as one-phonon excitations built on the ground state $|0\rangle$ as

$$
\begin{aligned}
& |i\rangle=\hat{\Gamma}_{i}^{\dagger}|0\rangle \\
& \hat{\Gamma}_{i}^{\dagger}=\sum_{\alpha \beta}\left\{f_{\alpha \beta}^{i} \hat{a}_{\alpha}^{\dagger} \hat{a}_{\beta}^{\dagger}-g_{\alpha \beta}^{i} \hat{a}_{\bar{\beta}} \hat{a}_{\bar{\alpha}}\right\},
\end{aligned}
$$

where $\hat{a}^{\dagger}$ and $\hat{a}$ are the quasiparticle (qp) creation and annihilation operators that are defined in terms of the solutions of the KSB equation (1) with the Bogoliubov transformation. The phonon states, the amplitudes $f^{i}, g^{i}$ and the vibrational frequency $\omega_{i}$, are obtained in the quasiparticle-random-phase approximation (QRPA): the linearized time-dependent density-functional theory for superfluid systems 29. The EDF gives the residual interactions entering into the QRPA equation. In the present calculation scheme, the QRPA equation is block diagonalized according to the quantum number $K=\Omega_{\alpha}+\Omega_{\beta}$.

\section{B. Numerical procedures}

I solve the KSB equation in the coordinate space using cylindrical coordinates $\boldsymbol{r}=(\varrho, z, \phi)$. Since I assume further the reflection symmetry, only the region of $z \geq 0$ is considered. I use a two-dimensional lattice mesh with $\varrho_{i}=(i-1 / 2) h, z_{j}=(j-1) h(i, j=1,2, \ldots)$ with a mesh size of $h=0.6 \mathrm{fm}$ and 25 points for each direction. The qp states are truncated according to the qp energy cutoff at $60 \mathrm{MeV}$, and the qp states up to the magnetic quantum number $\Omega=23 / 2$ with positive and negative parities are included. I introduce the truncation for the two-quasiparticle (2qp) configurations in the QRPA calculations, in terms of the 2qp-energy as $60 \mathrm{MeV}$.

For the normal (particle-hole) part of the EDF, I employ mainly the $\mathrm{SkM}^{*}$ functional [34, and use the SLy4 functional [35] to complement the discussion. For the pairing energy, I adopt the so-called mixed-type interaction:

$$
V_{\text {pair }}^{q}\left(\boldsymbol{r}, \boldsymbol{r}^{\prime}\right)=V_{0}^{q}\left[1-\frac{\rho(\boldsymbol{r})}{2 \rho_{0}}\right] \delta\left(\boldsymbol{r}-\boldsymbol{r}^{\prime}\right)
$$

with $\rho_{0}=0.16 \mathrm{fm}^{-3}$, and $\rho(\boldsymbol{r})$ being the isoscalar (matter) particle density. The same pair interaction is employed for the dynamical pairing in the QRPA calculation. The parameter $V_{0}^{q}$ is fitted to the three-point formula for the odd-even staggering centered at the oddmass system and averaged over the two neighboring nuclei [36, 37. In the present work, I fix the pairing parameters to reproduce reasonably the data of ${ }^{244} \mathrm{Cm}$ which are $0.63 \mathrm{MeV}$ and $0.57 \mathrm{MeV}$ for neutrons and protons, respectively. The resultant pairing gaps are $0.65 \mathrm{MeV}$ and $0.59 \mathrm{MeV}$ with $V_{0}^{\nu}=-270 \mathrm{MeV} \mathrm{fm}^{3}$ and $V_{0}^{\pi}=-310$ $\mathrm{MeV} \mathrm{fm}{ }^{3}$.

\section{RESULTS AND DISCUSSION}

\section{A. Ground-state properties}

Table[ summarizes the calculated deformation parameters:

$$
\beta_{2}^{q}=\frac{4 \pi}{5 N_{q}\left\langle r^{2}\right\rangle_{q}} \int \mathrm{d} \boldsymbol{r} Y_{20}(\hat{r}) \rho_{q}(\boldsymbol{r}),
$$


TABLE I. Calculated deformation parameters $\beta_{2}$. The evaluated data denoted as exp. are taken from Ref. 38. Listed in parentheses are the intrinsic quadrupole moments in the unit of $e \mathrm{~b}$ and $\mathrm{b}$ for protons and neutrons, respectively.

\begin{tabular}{lcccccc}
\hline \hline & \multicolumn{3}{c}{ protons } & & \multicolumn{2}{c}{ neutrons } \\
\cline { 2 - 4 } \cline { 5 - 6 } & SkM* & SLy4 & exp. & & SkM* & SLy4 \\
${ }^{244} \mathrm{Pu}$ & $0.29(11.7)$ & $0.28(11.5)$ & 0.29 & & $0.27(18.3)$ & $0.27(18.2)$ \\
${ }^{246} \mathrm{Cm}$ & $0.29(12.3)$ & $0.28(12.2)$ & 0.30 & & $0.27(18.7)$ & $0.27(18.6)$ \\
${ }^{248} \mathrm{Cf}$ & $0.30(12.9)$ & $0.30(12.9)$ & & $0.27(19.0)$ & $0.27(19.0)$ \\
${ }^{250} \mathrm{Fm}$ & $0.30(13.5)$ & $0.30(13.4)$ & & $0.28(19.3)$ & $0.28(19.2)$ \\
${ }^{252} \mathrm{No}$ & $0.30(13.9)$ & $0.30(13.7)$ & & $0.28(19.4)$ & $0.28(19.2)$ \\
\hline \hline
\end{tabular}

where the rms radius is given as $\sqrt{\left\langle r^{2}\right\rangle_{q}}=$ $\sqrt{\int r^{2} \rho_{q}(\boldsymbol{r}) \mathrm{d} \boldsymbol{r} / N_{q}}$, with $N_{q}$ being either the neutron number or proton number. They are compared with the experimental data 38, which is evaluated from the $B(\mathrm{E} 2)$ value based on the leading-order intensity relations of E2 matrix elements for an axially symmetric rotor 20]. The calculated results obtained by employing the SLy4 functional are also included. The strengths of the pair interaction were fitted similarly for $\mathrm{SkM}^{*}$; the strength $V_{0}^{\nu}=-310 \mathrm{MeV} \mathrm{fm}{ }^{3}$ and $V_{0}^{\pi}=-320 \mathrm{MeV} \mathrm{fm}^{3}$ produces the pair gap as $0.67 \mathrm{MeV}$ and $0.53 \mathrm{MeV}$ for neutrons and protons, respectively. Both the $\mathrm{SkM}^{*}$ and SLy4 functionals reproduce well the strong deformation of ${ }^{244} \mathrm{Pu}$ and ${ }^{246} \mathrm{Cm}$. The calculations predict a stronger deformation for higher $Z$ isotones: ${ }^{250} \mathrm{Fm}$ and ${ }^{252} \mathrm{No}$.

I show in Fig. 1 the pair gaps of protons and neutrons:

$$
\Delta^{q}=\left|\frac{\int \mathrm{d} \boldsymbol{r} \tilde{\rho}_{q}(\boldsymbol{r}) \tilde{U}^{q}(\boldsymbol{r})}{\int \mathrm{d} \boldsymbol{r} \tilde{\rho}_{q}(\boldsymbol{r})}\right|,
$$

where $\tilde{U}^{q}(\boldsymbol{r})$ is the pair potential given by $\tilde{h}_{\sigma \sigma^{\prime}}^{q}(\boldsymbol{r})=$ $\delta_{\sigma \sigma^{\prime}} \tilde{U}^{q}(\boldsymbol{r})$ and $\tilde{\rho}_{q}(\boldsymbol{r})$ is the anomalous (pair) density. The calculations overestimate the measurements for neutrons. When I slightly decrease the pair strength for neutrons as $V_{0}^{\nu}=-260 \mathrm{MeV} \mathrm{fm}^{3}$, the calculation shows a reasonable agreement. However, a proton-number dependence is opposite to what is shown in the measurements. This indicates that the neutrons deformed gap of 150 must be more significant than that of 148 in lower $Z$ and that the level density around $N=150$ is high in higher $Z$ nuclei. Since the deformed shell gap at 150 is formed by the up-sloping orbitals stemming from the $2 g_{9 / 2}$ shell and the down-sloping orbitals emanating from the $2 g_{7 / 2}$ shell, a slight modification of the spin-orbit interaction would impact the shell structure in this region, and further, the prediction of the magic numbers in the superheavy region, as anticipated in Ref. [8].

The resultant pair gaps of protons are compatible with the experimental ones. The $\mathrm{SkM}^{*}$ functional with the mixed-type pairing well reproduces the overall isotonic dependence, while the SLy4 shows a drop at $Z=98$, which the measurements do not reveal. One may conjecture that this is because the deformed shell gap is overdeveloped at $Z=98$ in the SLy4 model. I then show in

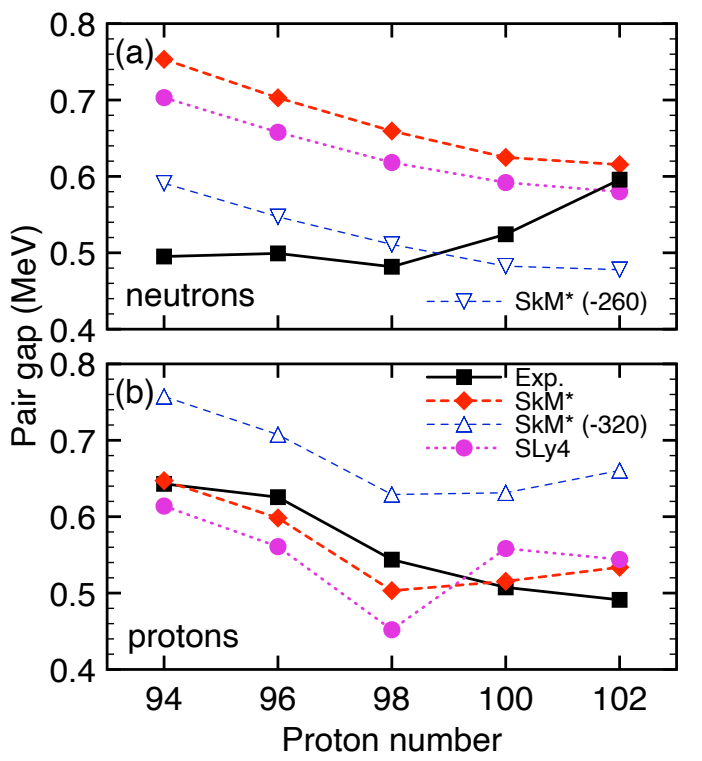

FIG. 1. Pair gaps of neutrons and protons in ${ }^{244} \mathrm{Pu},{ }^{246} \mathrm{Cm}$, ${ }^{248} \mathrm{Cf},{ }^{250} \mathrm{Fm}$, and ${ }^{252}$ No. The calculated gaps (filled diamonds) are compared with the experimental data (filled squares). The results obtained by reducing the pairing strength $V_{0}^{\nu}=-260 \mathrm{MeV} \mathrm{fm}^{3}$ while keeping the strength for protons, and those obtained by increasing the pairing strength $V_{0}^{\pi}=-320 \mathrm{MeV} \mathrm{fm}^{3}$ while keeping the strength for neutrons are also shown by open triangles for neutrons and protons, respectively. Depicted also are the results obtained by employing the SLy4 functional (filled circles).

Fig. 2 the single-particle levels in ${ }^{248} \mathrm{Cf}$ to visualize the shell structure. The single-particle orbitals were obtained by rediagonalizing the single-particle Hamiltonian:

$$
\sum_{\sigma^{\prime}} h_{\sigma \sigma^{\prime}}^{q}\left[\rho_{*}, \tilde{\rho}_{*}\right] \varphi_{i}^{q}\left(\boldsymbol{r} \sigma^{\prime}\right)=\varepsilon_{i}^{q} \varphi_{i}^{q}(\boldsymbol{r} \sigma)
$$

with $\rho_{*}$ and $\tilde{\rho}_{*}$ obtained as the solution of the KSB equation (1). The energy gap at $Z=98$ is indeed larger than that obtained by using the $\mathrm{SkM}^{*}$ functional, as discussed in Ref. 39. Since the pair gap is about $0.5 \mathrm{MeV}$, the orbitals with $\Omega^{\pi}=7 / 2^{+}$and $3 / 2^{-}$are primarily active for the pairing correlation. The occupation probability of the orbital with $\Omega^{\pi}=7 / 2^{+}$is indeed only 0.14 , and that of the orbital with $\Omega^{\pi}=3 / 2^{-}$is as large as 0.85 . For reference, they are 0.23 and 0.80 in the $\mathrm{SkM}^{*}$ model. A strong deformed-shell closure at $Z=98$ in the SLy4 model is unfavorable in generating the low-energy $K^{\pi}=2^{-}$state, as questioned in Ref. [18].

\section{B. Vibrational states}

I consider the response to the quadrupole $(\lambda=2)$ and octupole $(\lambda=3)$ operators defined by

$$
\hat{F}_{\lambda K}^{q}=\sum_{\sigma} \int \mathrm{d} \boldsymbol{r} r^{\lambda} Y_{\lambda K} \hat{\psi}_{q}^{\dagger}(\boldsymbol{r} \sigma) \hat{\psi}_{q}(\boldsymbol{r} \sigma),
$$



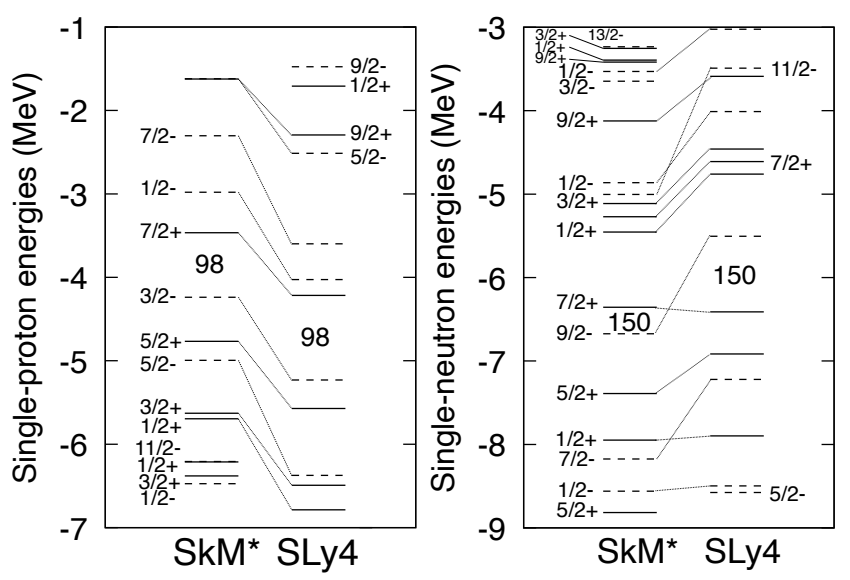

FIG. 2. Single-particle energies of protons and neutrons near the Fermi levels in ${ }^{248} \mathrm{Cf}$. The results obtained by using the SkM* and SLy4 functionals are compared. The solid and dashed lines indicate the positive- and negative-parity states, respectively. Each orbital is labeled by $\Omega^{\pi}$.

where $\hat{\psi}_{q}^{\dagger}(\boldsymbol{r} \sigma), \hat{\psi}_{q}(\boldsymbol{r} \sigma)$ represent the nucleon field operators. The reduced transition probabilities can be evaluated with the intrinsic transition strengths as

$$
\begin{aligned}
B\left(\mathrm{E} \lambda ; I_{K}^{\pi} \rightarrow 0_{\mathrm{gs}}^{+}\right) & =\frac{2-\delta_{K, 0}}{2 I_{K}+1} e^{2}\left|\left\langle i\left|\hat{F}_{\lambda K}^{\pi}\right| 0\right\rangle\right|^{2}, \\
B\left(\mathrm{IS} \lambda ; I_{K}^{\pi} \rightarrow 0_{\mathrm{gs}}^{+}\right) & =\frac{2-\delta_{K, 0}}{2 I_{K}+1}\left|\left\langle i\left|\hat{F}_{\lambda K}^{\pi}+\hat{F}_{\lambda K}^{\nu}\right| 0\right\rangle\right|^{2}
\end{aligned}
$$

in the rotational coupling scheme [20], where $I_{K}$ represents the nuclear spin of the $K^{\pi}$-band. The intrinsic transition matrix element is given as

$$
\begin{aligned}
\left\langle i\left|\hat{F}_{\lambda K}^{q}\right| 0\right\rangle & =\sum_{\alpha \beta}\left(f_{\alpha \beta}^{i}+g_{\alpha \beta}^{i}\right)\left\langle\alpha \beta\left|\hat{F}_{\lambda K}^{q}\right| 0\right\rangle \\
& \equiv \sum_{\alpha \beta} M_{\alpha \beta}^{q, i}
\end{aligned}
$$

with the QRPA amplitudes and the 2qp matrix elements. The QRPA amplitudes are normalized as

$$
\sum_{\alpha \beta}\left[\left|f_{\alpha \beta}^{i}\right|^{2}-\left|g_{\alpha \beta}^{i}\right|^{2}\right]=1
$$

$$
\text { 1. } K^{\pi}=2^{-} \text {state }
$$

I show in Fig. 3(a) the intrinsic excitation energies of the $K^{\pi}=2^{-}$state in the $N=150$ isotones. Both the $\mathrm{SkM}^{*}$ and SLy4 functionals well reproduce the isotonic trend of the energy, particularly a drop at $Z=98$, but overestimate the measurements as a whole. The QRPA calculation in Ref. [18] based on the Nilsson potential [40] also well describes a sharp fall in energies at $Z=98$, while the one employing the D1M functional shows only a smooth proton-number dependence. It is noted that

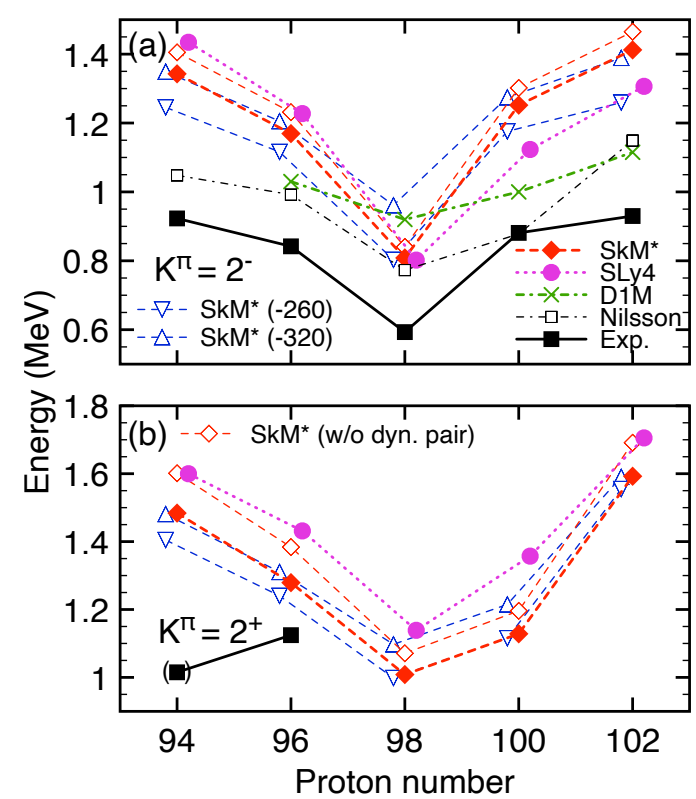

FIG. 3. Excitation energies of the (a) $K^{\pi}=2^{-}$and (b) $K^{\pi}=2^{+}$states in the $N=150$ isotones. The calculations employing the SkM* and SLy4 functionals are compared with the measurements [18, 22, 23. The results obtained by increasing the pairing for protons $\left(V_{0}^{\pi}=-320 \mathrm{MeV} \mathrm{fm}^{3}\right)$ and decreasing the pairing for neutrons $\left(V_{0}^{\nu}=-260 \mathrm{MeV} \mathrm{fm}^{3}\right)$ are depicted by open triangles. The $\mathrm{SkM}^{*}$ results without the dynamical pairing are given by open diamonds. Shown is also the calculations using the Gogny D1M functional [19] and the Nilsson potential [18.

in these calculations, the rotational correction and the Coriolis coupling effect [41] are not included. One needs to consider these effects for a quantitative description, but it is beyond the scope of the present study. Rather, I am going to investigate a microscopic mechanism for the unique isotonic dependence of the octupole correlation.

A lowering in energy implies that the collectivity develops from $Z=96$ to $Z=98$. However, the isoscalar intrinsic transition strengths to the $K^{\pi}=2^{-}$state do not show such a trend: $6.83,6.18,6.11,4.74$, and 4.45 (in $10^{5}$ $\mathrm{fm}^{6}$ ) in ${ }^{244} \mathrm{Pu},{ }^{246} \mathrm{Cm},{ }^{248} \mathrm{Cf},{ }^{250} \mathrm{Fm}$, and ${ }^{252}$ No. The isotonic dependence of the properties of the $K^{\pi}=2^{-}$state is actually governed by the details around the Fermi surface: the interplay between the underlying shell structures and the pairing correlations. To see the role of the pairing, shown in Fig. 3(a) are the results obtained by varying the pair interaction. I reduced the pairing strength $V_{0}^{\nu}=-260 \mathrm{MeV} \mathrm{fm}{ }^{3}$ while keeping the strength for protons, and I increased the pairing strength $V_{0}^{\pi}=-320 \mathrm{MeV} \mathrm{fm}^{3}$ while keeping the strength for neutrons. Then, I find that the $K^{\pi}=2^{-}$state in ${ }^{248} \mathrm{Cf}$ is sensitive to the pairing of protons. When the strength of the pair interaction for protons is increased, the excitation energy in ${ }^{248} \mathrm{Cf}$ becomes higher: $0.84 \mathrm{MeV}$ to $1.00 \mathrm{MeV}$, while the excitation energy in the other iso- 


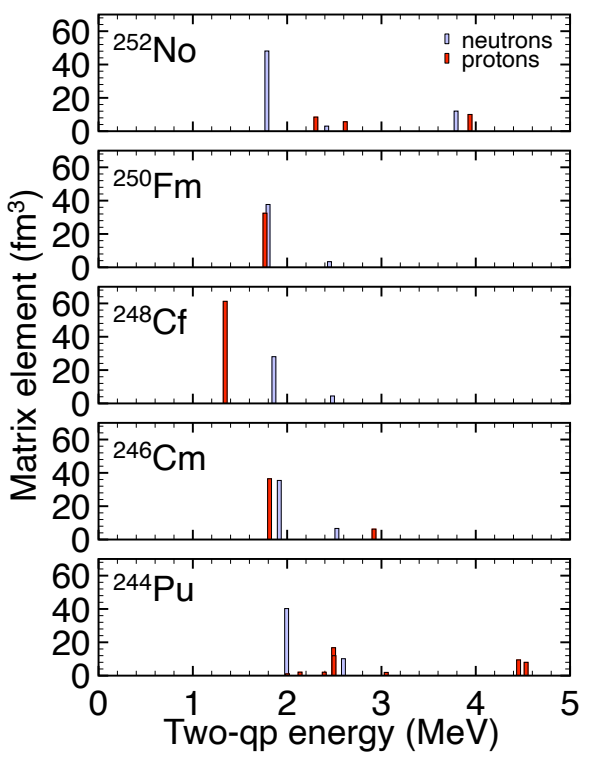

FIG. 4. Matrix elements $M_{\alpha \beta}$ 12 of the 2qp excitations near the Fermi levels for the $K^{\pi}=2^{-}$state as functions of the unperturbed 2qp energy $E_{\alpha}+E_{\beta}$.

tones does not change much. As seen in Fig. 1(b), $2 \Delta$ for protons rises by about $0.2 \mathrm{MeV}$ in increasing the pairing strength for protons: the increase in the pairing gap of protons directly affects the increase in the excitation energy. On the other hand, the change in the pairing strength for neutrons does not alter the energy in ${ }^{248} \mathrm{Cf}$. This indicates that the $K^{\pi}=2^{-}$state is sensitive to the excitation of protons. The calculation in Ref. [18. found a high amplitude for the proton configuration of $[633] 7 / 2 \otimes[521] 3 / 2$, and the quasiparticle phonon model predicted that the main component of the wave function is this proton configuration with a weight of $62 \%$ [2]. Containing a large component of this proton configuration is supported by the population of the $K^{\pi}=2^{-}$and $5^{-}$states in the ${ }^{249} \mathrm{Bk}(\alpha, \mathrm{t}){ }^{250} \mathrm{Cf}$ reaction [43].

Let me investigate the isotonic evolution of the microscopic structure. Figure 4 shows the matrix elements $M_{\alpha \beta}$ defined in Eq. 12 of the 2qp excitations near the Fermi levels for the $\bar{K}^{\pi}=2^{-}$state. One can see that 2qp excitations near the Fermi levels have a coherent contribution to generate the $K^{\pi}=2^{-}$state with the same sign. It should be noted that not only the 2qp excitations near the Fermi levels but those in the giant-resonance region have a coherent contribution for the enhancement in the transition strength to the low-frequency mode, as discussed in Ref. [27. In ${ }^{244} \mathrm{Pu}$, the neutron 2qp

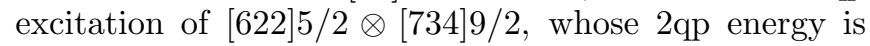
about $2 \mathrm{MeV}$, is a main component with an amplitude $|f|^{2}-|g|^{2}$ of 0.45 . This is why the decrease in the pairing strength for neutrons lowers the excitation energy. When adding two protons to ${ }^{244} \mathrm{Pu}$, the Fermi level of protons becomes higher. The proton 2qp excitation of $[521] 3 / 2 \otimes[633] 7 / 2$, whose 2 qp energy decreases to 1.8
$\mathrm{MeV}$, then has an appreciable contribution with an amplitude of 0.43 , together with the $\nu[622] 5 / 2 \otimes \nu[734] 9 / 2$ excitation with an amplitude of 0.31 . Note that both the $\pi[521] 3 / 2$ and $\pi[633] 7 / 2$ orbitals are particle-like. In ${ }^{248} \mathrm{Cf}$, the Fermi level of protons is located just in between the $\pi[521] 3 / 2$ and $\pi[633] 7 / 2$ orbitals. Then, the 2qp excitation of $\pi[521] 3 / 2 \otimes \pi[633] 7 / 2$ appears low in energy at $1.3 \mathrm{MeV}$, and the $K^{\pi}=2^{-}$state is predominantly generated by this configuration with an amplitude of 0.72 . The amplitude of the $\nu[622] 5 / 2 \otimes \nu[734] 9 / 2$ excitation declines to 0.14 . Adding two more protons, the 2qp excitation of $\pi[521] 3 / 2 \otimes \pi[633] 7 / 2$ is a hole-hole type excitation, and the unperturbed 2qp states are located higher in energy. Therefore, the matrix element and contribution of this 2qp excitation decrease. The $K^{\pi}=2^{-}$state in ${ }^{250} \mathrm{Fm}$ is mainly constructed by the $\pi[521] 3 / 2 \otimes \pi[633] 7 / 2$ and $\nu[622] 5 / 2 \otimes \nu[734] 9 / 2$ excitations with a wight of 0.42 and 0.41 , respectively. Then, the $\nu[622] 5 / 2 \otimes \nu[734] 9 / 2$ excitation dominantly generates the $K^{\pi}=2^{-}$state with a weight of 0.73 in ${ }^{252}$ No.

It seems that the collectivity of the $K^{\pi}=2^{-}$state in ${ }^{248} \mathrm{Cf}$ is weaker than in the other isotones: single $2 \mathrm{qp}$ excitation dominates the state. The sum of the backwardgoing amplitudes $\sum|g|^{2}$ and the octupole polarizability $\chi$ can be measures of the correlation, in addition to the energy shift and the enhancement in the transition strength due to the QRPA correlations. The calculated $\sum|g|^{2}$ is $0.22,0.20,0.24,0.14$, and 0.12 and the stiffness parameter $C_{\beta_{32}}=\chi_{\beta_{32}}^{-1}$ (in $\mathrm{MeV}$ ) is 418, 402, 306, 502, and 548 in ${ }^{244} \mathrm{Pu},{ }^{246} \mathrm{Cm},{ }^{248} \mathrm{Cf},{ }^{250} \mathrm{Fm}$, and ${ }^{252} \mathrm{No}$, respectively. Based on these measures, the octupole correlation is strong in ${ }^{248} \mathrm{Cf}$ as well as in ${ }^{244} \mathrm{Pu}$ and ${ }^{246} \mathrm{Cm}$.

In Fig. 3(a), I plot the calculated results obtained by neglecting the dynamical pairing, as depicted by open diamonds. The dynamical pairing lowers the $K^{\pi}=2^{-}$ state by $0.04-0.06 \mathrm{MeV}$. One sees a larger effect in ${ }^{246} \mathrm{Cm}$. The energy shift due to the dynamical pairing is 0.06 $\mathrm{MeV}$ out of $0.65 \mathrm{MeV}$ due to the QRPA correlations in total. It is noted that the pairing correlation energy in the total binding energy is about $0.7 \%$. As discussed above, the collective state in ${ }^{246} \mathrm{Cm}$ is constructed mainly by the $\nu[622] 5 / 2 \otimes \nu[734] 9 / 2$ and $\pi[521] 3 / 2 \otimes \pi[633] 7 / 2$ excitations. Since these are a neutron hole-hole and a proton particle-particle excitations, the residual pair interaction for both neutrons and protons is active and instrumental in forming the collective state.

Surprisingly, the SLy4 model reproduces the drop in energies at $Z=98$. As mentioned above, and as questioned in Ref. [18, Fig. 2 shows that the SLy4 model overestimates the deformed gap at $Z=98$, requiring a high energy particle-hole excitation from $\pi[521] 3 / 2$ to $\pi[633] 7 / 2$. Here, the pairing correlations are key to understanding the characteristic isotonic dependence. The microscopic structure of the $K^{\pi}=2^{-}$state in the SLy4 model is essentially the same as in the $\mathrm{SkM}^{*}$ model: the $\nu[622] 5 / 2 \otimes \nu[734] 9 / 2$ and $\pi[521] 3 / 2 \otimes \pi[633] 7 / 2$ excitations play a central role. The unperturbed energy of the $\pi[521] 3 / 2 \otimes \pi[633] 7 / 2$ excitation is $1.93 \mathrm{MeV}$ and 1.41 
$\mathrm{MeV}$ in ${ }^{246} \mathrm{Cm}$ and ${ }^{248} \mathrm{Cf}$; a $0.5 \mathrm{MeV}$ drop is comparable to the calculation in the $\mathrm{SkM}^{*}$ model, as shown in Fig. 4 . The reduction of the pairing of protons, associated with a strong shell closure, accounts for the drop in energy.

As one sees in Fig. 2, the SkM* and SLy4 functionals give different shell structures of neutrons around $N=150$ : the single-particle orbitals with $\Omega^{\pi}=7 / 2^{+}$and $9 / 2^{-}$are inverted. Therefore, the $\nu[622] 5 / 2 \otimes \nu[734] 9 / 2$ excitation is a hole-hole type excitation in the $\mathrm{SkM}^{*}$ model, and this becomes a particle-hole type in $N=148$. One can thus expect that the collectivity of the $K^{\pi}=2^{-}$ state is strongest in ${ }^{246} \mathrm{Cf}$. Indeed, the $\mathrm{SkM}^{*}$ model gives a strongly collective $K^{\pi}=2^{-}$state at $0.65 \mathrm{MeV}$, with the isoscalar strength of $7.55 \times 10^{5} \mathrm{fm}^{6}$, which is predominantly generated by the $\nu[622] 5 / 2 \otimes \nu[734] 9 / 2$ excitation with a weight of 0.31 and the $\pi[521] 3 / 2 \otimes \pi[633] 7 / 2$ excitation with a weight of 0.56 . The sum of backward-going amplitudes is 0.35 , and the stiffness parameter is 218 $\mathrm{MeV}$. If the $K^{\pi}=2^{-}$state is observed in a future experiment at lower energy than in ${ }^{248} \mathrm{Cf}$, our understanding of the shell structure around $N=150$ will be greatly deepened. The ground state in ${ }^{249} \mathrm{Cf}$ is $\nu[734] 9 / 2$ and the excited bands of the $\nu[622] 5 / 2$ and $\nu[624] 7 / 2$ appear at $145 \mathrm{keV}$ and $380 \mathrm{keV}$, respectively [44] it suggests the ordering of the single-particle levels as given in the $\mathrm{SkM}^{*}$ model though the deformed gap of $N=150$ must be larger than that of 148 .

$$
\text { 2. } K^{\pi}=2^{+} \text {state }
$$

I show in Fig. 3(b) the intrinsic excitation energies of the $K^{\pi}=2^{+}$state in the $N=150$ isotones. The excitation energy calculated is higher than that of a typical $\gamma$ vibration: experimentally, the $2_{2}^{+}$states were observed at low excitation energies $(\sim 800 \mathrm{keV})$ for the well-deformed dysprosium $(Z=66)$ and erbium $(Z=68)$ isotopes around $N=98$ [45] and in the neutron-rich region [46, 47. It is noted that the present nuclear EDF method well describes the $\gamma$ vibration in the neutron-rich Dy isotopes [4].

One can see a similar isotonic dependence of the excitation energy to the $K^{\pi}=2^{-}$state: a drop at around $Z=98$. As for the $K^{\pi}=2^{-}$state, this is governed by the underlying shell structure. Let me then investigate the isotonic evolution of the microscopic structure. Figure 5 shows the matrix elements $M_{\alpha \beta}$ defined in Eq. 12 of the 2qp excitations near the Fermi levels for the $\bar{K}^{\pi}=2^{+}$state. One sees that 2qp excitations near the Fermi levels have a coherent contribution to generate the $K^{\pi}=2^{+}$state with the same sign as for the $K^{\pi}=2^{-}$ state. Table II summarizes the amplitudes $|f|^{2}-|g|^{2}$ for the configurations possessing a dominant contribution to the $K^{\pi}=2^{+}$state. Both the neutron 2qp excitations A: $[620] 1 / 2 \otimes[622] 5 / 2$ and B: [622] $3 / 2 \otimes[624] 7 / 2$ satisfy the selection rule of the $\gamma$ vibration [20]:

$$
\Delta N=0 \text { or } 2, \Delta n_{3}=0, \Delta \Lambda=\Delta \Omega= \pm 2 .
$$

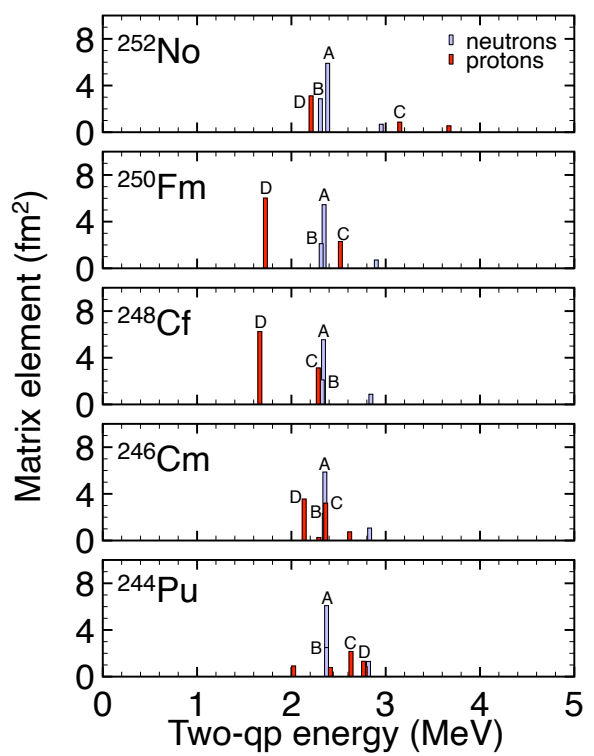

FIG. 5. Similar to Fig. 4 but for the $K^{\pi}=2^{+}$state. See Tab. II for the configurations A, B, C, and D.

TABLE II. Amplitudes $|f|^{2}-|g|^{2}$ of the 2qp excitations possessing a dominant contribution to the $K^{\pi}=2^{+}$state.

\begin{tabular}{lccccc}
\hline \hline configuration & ${ }^{244} \mathrm{Pu}$ & ${ }^{246} \mathrm{Cm}$ & ${ }^{248} \mathrm{Cf}$ & ${ }^{250} \mathrm{Fm}$ & ${ }^{252} \mathrm{No}$ \\
\hline A: $\nu[620] 1 / 2 \otimes \nu[622] 5 / 2$ & 0.31 & 0.27 & 0.18 & 0.20 & 0.30 \\
B: $\nu[622] 3 / 2 \otimes \nu[624] 7 / 2$ & 0.14 & 0.11 & 0.07 & 0.08 & 0.20 \\
C: $\pi[521] 1 / 2 \otimes \pi[512] 5 / 2$ & 0.12 & 0.18 & 0.14 & 0.09 & 0.03 \\
D: $\pi[521] 1 / 2 \otimes \pi[521] 3 / 2$ & 0.07 & 0.27 & 0.49 & 0.52 & 0.32 \\
\hline \hline
\end{tabular}

However, the matrix element for B is small as this configuration is a particle-particle excitation.

The proton 2qp excitation D: [521]1/2 $\otimes[521] 3 / 2$ governs the isotonic dependence of the $K^{\pi}=2^{+}$state, that satisfies the selection rule as well. The $\pi[521] 3 / 2$ orbital is located above the Fermi level of protons in ${ }^{244} \mathrm{Pu}$ and ${ }^{246} \mathrm{Cm}$, and the $\pi[521] 1 / 2$ orbital is located below the Fermi level in ${ }^{252}$ No, as seen in Fig. 2 Thus, the transition matrix element of the 2qp excitation D is small in these isotones. The proton 2qp excitation C: $[521] 1 / 2 \otimes[512] 5 / 2$ is a particle-hole excitation in $Z=94-100$, and this has an appreciable contribution in the isotones under the present study. However, the particle-hole energy is relatively high, and the matrix element is not significant because the excitation is in disagreement with the selection rule. In this respect, ${ }^{248} \mathrm{Cf}$ and ${ }^{250} \mathrm{Fm}$ are the most favorable systems where the $\gamma$ vibration occurs in the low energy. The excitation energy of the $K^{\pi}=2^{+}$state is higher than the $K^{\pi}=2^{-}$ state. This is because the quasi-proton [521]1/2 orbital is higher in energy than the [633]7/2 orbital. However, the $\mathrm{SkM}^{*}$ model predicts that the $K^{\pi}=2^{+}$state appears lower than the $K^{\pi}=2^{-}$state in ${ }^{250} \mathrm{Fm}$; this may be in contradiction with the measurements of the low-lying 
states in ${ }^{251} \mathrm{Md}$ [49], where the ground state is suggested to be $\pi[514] 7 / 2$ and the excited band of the $\pi[521] 1 / 2$ configuration appears at $55 \mathrm{keV}$ as the lowest state.

Finally, I investigate the roles of the pairing correlations. Plotted in Fig. 3(b) are the results obtained by decreasing the pairing strength for neutrons (open triangles-down) and by increasing the pairing strength for protons (open triangles-up). One sees that the decrease of the pairing for neutrons lowers the excitation energies, and the effect is larger in the $\mathrm{Pu}, \mathrm{Cm}$, and $\mathrm{No}$ isotones. The increase of the pairing for protons raises the excitation energies in the $\mathrm{Cf}$ and $\mathrm{Fm}$ isotones. As the increase (decrease) of the pairing strength enhances (reduces) the pairing gap, the QRPA frequencies rise (decline). The pairing effect depends on the structure of the states. To see one aspect of the structure, let me introduce the quantity: $\left|M_{\nu} / M_{\pi}\right|=\left|\left\langle i\left|\hat{F}^{\nu}\right| 0\right\rangle /\left\langle i\left|\hat{F}^{\pi}\right| 0\right\rangle\right|$. For the $K^{\pi}=2^{+}$states calculated in the SkM* model, this is $0.93,0.89,0.85,0.86$, and 0.98 in ${ }^{244} \mathrm{Pu},{ }^{246} \mathrm{Cm},{ }^{248} \mathrm{Cf}$, ${ }^{250} \mathrm{Fm}$, and ${ }^{252} \mathrm{No}$, respectively. Here, the $\left|M_{\nu} / M_{\pi}\right|$ value is divided by $N / Z$. The excitation of protons is stronger in the Cf and Fm, which is in accordance with the microscopic structure discussed above. The pairing of neutrons (protons) is thus relatively sensitive in the $\mathrm{Pu}, \mathrm{Cm}, \mathrm{No}$ (Cf, Fm) isotones. The dynamical pairing enhances the collectivity: the energy shift due to the residual pair interaction is $0.06 \mathrm{MeV}-0.12 \mathrm{MeV}$. This is equal to, but more substantial than, the case in the $K^{\pi}=2^{-}$state, indicating that the coherence among particle-hole and particle-particle (hole-hole) excitations takes place more strongly than in the $K^{\pi}=2^{-}$state.

\section{SUMMARY}

I have investigated the microscopic mechanism for the appearance of a low-energy $K^{\pi}=2^{-}$state in the $N=150$ isotones in the actinides, in particular, a sharp drop in energy in ${ }^{248} \mathrm{Cf}$. Furthermore, I have studied the possible occurrence of the low-energy $K^{\pi}=2^{+}$state to elucidate the mechanism that prefers the simultaneous breaking of the reflection and axial symmetry to the breaking of the axial symmetry alone in this mass region. To this end, I employed the nuclear energydensity functional (EDF) method: the Skyrme-KohnSham-Bogoliubov and the quasiparticle random-phase approximation were used to describe the ground state and the transition to excited states.

The Skyrme-type SkM* and SLy4 functionals reproduce the fall in the energy of the $K^{\pi}=2^{-}$state at $Z=98$, where the proton two-quasiparticle (2qp) excitation $[633] 7 / 2 \otimes[521] 3 / 2$ plays a decisive role for the peculiar isotonic dependence. I have found interweaving roles by the pairing correlations of protons and the deformed shell closure at $Z=98$ formed by the $[633] 7 / 2$ and [521]3/2 orbitals: the SLy4 model produces a strong shell closure, and accordingly, the pairing of protons is greatly suppressed, resulting in a drop in energy. The $\mathrm{SkM}^{*}$ model predicts that the $K^{\pi}=2^{-}$state appears lower in energy in ${ }^{246} \mathrm{Cf}$ than in ${ }^{248} \mathrm{Cf}$ as the Fermi level of neutrons is located in between the [622]5/2 and [734] $9 / 2$ orbitals.

The $K^{\pi}=2^{+}$state is predicted to appear higher in energy than the $K^{\pi}=2^{-}$state as the quasi-proton orbital [521]1/2 is located above the $[633] 7 / 2$ orbital, except for ${ }^{250} \mathrm{Fm}$ in the $\mathrm{SkM}^{*}$ model with being unlikely based on the spectroscopic study of ${ }^{251} \mathrm{Md}$. Compared with the available experimental data, the EDFs presently used are not perfect at quantitatively describing pair correlations and shell structures. The present study shows a need for improvements in the EDFs based on further comparative studies for describing pair correlations and shell structures in heavy nuclei, that are indispensable in predicting the shell effect and stability of superheavy nuclei.

\section{ACKNOWLEDGMENTS}

This work was supported by the JSPS KAKENHI (Grants No. JP19K03824 and No. JP19K03872). The numerical calculations were performed on Yukawa-21 at the Yukawa Institute for Theoretical Physics, Kyoto University.
[1] A. Sobiczewski and K. Pomorski, Description of structure and properties of superheavy nuclei, Prog. Part. Nucl. Phys. 58, 292 (2007).

[2] Y. T. Oganessian and V. K. Utyonkov, Super-heavy element research, Rep. Prog. Phys. 78, 036301 (2015)

[3] G. G. Adamian, N. V. Antonenko, H. Lenske, L. A. Malov, and S.-G. Zhou, Self-consistent methods for structure and production of heavy and superheavy nuclei, Eur. Phys. J. A 57, 89 (2021)

[4] S. Karatzikos, A. V. Afanasjev, G. A. Lalazissis, and P. Ring, The Fission barriers in Actinides and superheavy nuclei in covariant density functional theory, Phys. Lett. B 689, 72 (2010), arXiv:0909.1233 [nucl-th]
[5] D. Ackermann and C. Theisen, Nuclear structure features of very heavy and superheavy nuclei-tracing quantum mechanics towards the 'island of stability', Phys. Scr. 92, $083002(2017)$

[6] R.-D. Herzberg and P. Greenlees, In-beam and decay spectroscopy of transfermium nuclei, Prog. Part. Nucl. Phys. 61, 674 (2008).

[7] C. Theisen, P. Greenlees, T.-L. Khoo, P. Chowdhury, and T. Ishii, In-beam spectroscopy of heavy elements, Nucl. Phy. A 944, 333 (2015), Special Issue on Superheavy Elements.

[8] M. Bender, P. Bonche, T. Duguet, and P. H. Heenen, Skyrme mean field study of rotational bands in 
transfermium isotopes, Nucl. Phys. A 723, 354 (2003) arXiv:nucl-th/0302058

[9] T. Duguet, P. Bonche, and P. H. Heenen, Rotational properties of ${ }^{252,253,254} \mathrm{No}$ : influence of pairing correlations, Nucl. Phys. A 679, 427 (2001), arXiv:nuclth/0005040

[10] A. V. Afanasjev, T. L. Khoo, S. Frauendorf, G. A. Lalazissis, and I. Ahmad, Cranked relativistic HartreeBogoliubov theory: Probing the gateway to superheavy nuclei, Phys. Rev. C 67, 024309 (2003), arXiv:nuclth/0301008

[11] S. K. Tandel, T. L. Khoo, D. Seweryniak, G. Mukherjee, I. Ahmad, B. Back, R. Blinstrup, M. P. Carpenter, J. Chapman, P. Chowdhury, C. N. Davids, A. A. Hecht, A. Heinz, P. Ikin, R. V. F. Janssens, F. G. Kondev, T. Lauritsen, C. J. Lister, E. F. Moore, D. Peterson, P. Reiter, U. S. Tandel, X. Wang, and S. Zhu, $K$ Isomers in ${ }^{254}$ No: Probing Single-Particle Energies and Pairing Strengths in the Heaviest Nuclei, Phys. Rev. Lett. 97, 082502 (2006)

[12] P. T. Greenlees, J. Rubert, J. Piot, B. J. P. Gall, L. L. Andersson, M. Asai, Z. Asfari, D. M. Cox, F. Dechery, O. Dorvaux, T. Grahn, K. Hauschild, G. Henning, A. Herzan, R.-D. Herzberg, F. P. Heßberger, U. Jakobsson, P. Jones, R. Julin, S. Juutinen, S. Ketelhut, T.L. Khoo, M. Leino, J. Ljungvall, A. Lopez-Martens, R. Lozeva, P. Nieminen, J. Pakarinen, P. Papadakis, E. Parr, P. Peura, P. Rahkila, S. Rinta-Antila, P. Ruotsalainen, M. Sandzelius, J. Sarén, C. Scholey, D. Seweryniak, J. Sorri, B. Sulignano, C. Theisen, J. Uusitalo, and M. Venhart, Shell-Structure and Pairing Interaction in Superheavy Nuclei: Rotational Properties of the $Z=104$ Nucleus ${ }^{256}$ Rf, Phys. Rev. Lett. 109, 012501 (2012)

[13] A. V. Afanasjev and O. Abdurazakov, Pairing and rotational properties of actinides and superheavy nuclei in covariant density functional theory, Phys. Rev. C 88, 014320 (2013), arXiv:1307.4131 [nucl-th]

[14] S. W. Yates, R. R. Chasman, A. M. Friedman, I. Ahmad, and K. Katori, Particle-phonon interactions in ${ }^{248} \mathrm{Cf}$ and ${ }^{249}$ Cf, Phys. Rev. C 12, 442 (1975)

[15] S. W. Yates, A. M. Friedman, and I. Ahmad, Octupole states in ${ }^{246} \mathrm{Cm}$ and ${ }^{248} \mathrm{Cm}$ populated by inelastic deuteron scattering, Phys. Rev. C 12, 795 (1975).

[16] Y. S. Chen, Y. Sun, and Z.-C. Gao, Nonaxial-octupole effect in superheavy nuclei, Phys. Rev. C 77, 061305 (2008), arXiv:0806.1983 [nucl-th].

[17] J. Zhao, B.-N. Lu, E.-G. Zhao, and S.-G. Zhou, Nonaxialoctupole $Y_{32}$ correlations in $N=150$ isotones from multidimensional constrained covariant density functional theories, Phys. Rev. C 86, 057304 (2012), arXiv:1209.6567 [nucl-th]

[18] A. P. Robinson, T. L. Khoo, I. Ahmad, S. K. Tandel, F. G. Kondev, T. Nakatsukasa, D. Seweryniak, M. Asai, B. B. Back, M. P. Carpenter, P. Chowdhury, C. N. Davids, S. Eeckhaudt, J. P. Greene, P. T. Greenlees, S. Gros, A. Heinz, R.-D. Herzberg, R. V. F. Janssens, G. D. Jones, T. Lauritsen, C. J. Lister, D. Peterson, J. Qian, U. S. Tandel, X. Wang, and S. Zhu, $K^{\pi}=8^{-}$ isomers and $K^{\pi}=2^{-}$octupole vibrations in $N=150$ shell-stabilized isotones, Phys. Rev. C 78, 034308 (2008).

[19] K. Rezynkina, A. Lopez-Martens, K. Hauschild, I. Deloncle, S. Péru, P. Brionnet, M. L. Chelnokov, V. I. Chepigin, O. Dorvaux, F. Déchery, H. Faure, B. Gall, A. V. Isaev, I. N. Izosimov, D. E. Katrasev, A. N. Kuznetsov,
A. A. Kuznetsova, O. N. Malyshev, A. G. Popeko, Y. A. Popov, E. A. Sokol, A. I. Svirikhin, and A. V. Yeremin, Influence of octupole vibration on the low-lying structure of ${ }^{251} \mathrm{Fm}$ and other heavy $N=151$ isotones, Phys. Rev. C 97, 054332 (2018).

[20] A. Bohr and B. Mottelson, Nuclear Structure: Volume II, Nuclear Deformations (Benjamin, 1969).

[21] P. Ring and P. Schuck, The nuclear many-body problem (Springer-Verlag, New York, 1980).

[22] L. G. Multhauf, K. G. Tirsell, R. J. Morrow, and R. A. Meyer, Levels of ${ }^{246} \mathrm{Cm}$ from the $\beta^{-}$-Decay Sequence ${ }^{246} \mathrm{Pu}(11$ days $){ }^{246 m} \mathrm{Am}(25 \mathrm{~min}){ }^{246} \mathrm{Cm}$, Phys. Rev. C 3, 1338 (1971).

23] R. C. Thompson, J. R. Huizenga, and T. W. Elze, Collective states in ${ }^{230} \mathrm{Th},{ }^{240} \mathrm{Pu},{ }^{244} \mathrm{Pu}$, and ${ }^{248} \mathrm{Cm}$ excited by inelastic deuteron scattering, Phys. Rev. C 12, 1227 (1975)

[24] K. Matsuyanagi, N. Hinohara, and K. Sato, BCS-Pairing and Nuclear Vibrations, Fifty Years of Nuclear BCS, 111-124 (2013), arXiv:1205.0078 [nucl-th]

[25] K. Yoshida, M. Yamagami, and K. Matsuyanagi, Dynamic pairing effects on low-frequency modes of excitation in deformed $\mathrm{Mg}$ isotopes close to the neutron drip line, Phys. Scr. T 125, 45 (2006), arXiv:nucl-th/0507047

[26] K. Yoshida, M. Yamagami, and K. Matsuyanagi, Pairing and continuum effects on low-frequency quadrupole vibrations in deformed $\mathrm{Mg}$ isotopes close to the neutron drip line, Nucl. Phys. A 779, 99 (2006), arXiv:nuclth/0605073

[27] K. Yoshida and M. Yamagami, Low-frequency $K^{\pi}=0^{+}$ modes in deformed neutron-rich nuclei: Pairing- and $\beta$ vibrational modes of neutron, Phys. Rev. C 77, 044312 (2008), arXiv:0802.2341 [nucl-th].

[28] M. Bender, P.-H. Heenen, and P.-G. Reinhard, Selfconsistent mean-field models for nuclear structure, Rev. Mod. Phys. 75, 121 (2003)

[29] T. Nakatsukasa, K. Matsuyanagi, M. Matsuo, and K. Yabana, Time-dependent density-functional description of nuclear dynamics, Rev. Mod. Phys. 88, 045004 (2016), arXiv:1606.04717

[30] K. Yoshida and N. Van Giai, Deformed quasiparticlerandom-phase approximation for neutron-rich nuclei using the Skyrme energy density functional, Phys. Rev. C 78, 064316 (2008), arXiv:0809.0169 [nucl-th].

[31] K. Yoshida and T. Nakatsukasa, Shape evolution of giant resonances in Nd and Sm isotopes, Phys. Rev. C 88, 034309 (2013), arXiv:1305.6437 [nucl-th]

[32] J. Dobaczewski, H. Flocard, and J. Treiner, HartreeFock-Bogolyubov description of nuclei near the neutrondrip line, Nucl. Phys. A 422, 103 (1984).

[33] H. Kasuya and K. Yoshida, Hartree-Fock-Bogoliubov theory for odd-mass nuclei with a time-odd constraint and application to deformed halo nuclei, Prog. Theor. Exp. Phys. 2021, 013D01 (2021), arXiv:2005.03276 [nuclth]

[34] J. Bartel, P. Quentin, M. Brack, C. Guet, and H.-B. Håkansson, Towards a better parametrisation of Skyrmelike effective forces: A critical study of the SkM force, Nucl. Phys. A 386, 79 (1982).

[35] E. Chabanat, P. Bonche, P. Haensel, J. Meyer, and R. Schaeffer, A Skyrme parametrization from subnuclear to neutron star densities. 2. Nuclei far from stablities, Nucl. Phys. A 635, 231 (1998), [Erratum: Nucl.Phys.A 643, 441-441 (1998)]. 
[36] W. Satuła, J. Dobaczewski, and W. Nazarewicz, Oddeven staggering of nuclear masses: pairing or shape effect?, Phys. Rev. Lett. 81, 3599 (1998), arXiv:nuclth/9804060

[37] J. Dobaczewski and W. Nazarewicz, Mean-field and pairing properties of exotic nuclei: Exploring the nuclear landscape, Prog. Theor. Phys. Suppl. 146, 70 (2002).

[38] B. Pritychenko, M. Birch, B. Singh, and M. Horoi, Tables of E2 Transition Probabilities from the first $2^{+}$States in Even-Even Nuclei, Atom. Data Nucl. Data Tabl. 107, 1 (2016), [Erratum: Atom.Data Nucl.Data Tabl. 114, 371374 (2017)], arXiv:1312.5975 [nucl-th]

[39] A. Chatillon, C. Theisen, P. T. Greenlees, G. Auger, J. E. Bastin, E. Bouchez, B. Bouriquet, J. M. Casandjian, R. Cee, E. Clément, R. Dayras, G. de France, R. de Toureil, S. Eeckhaudt, A. Görgen, T. Grahn, S. Grévy, K. Hauschild, R. D. Herzberg, P. J. C. Ikin, G. D. Jones, P. Jones, R. Julin, S. Juutinen, H. Kettunen, A. Korichi, W. Korten, Y. Le Coz, M. Leino, A. Lopez-Martens, S. M. Lukyanov, Y. E. Penionzhkevich, J. Perkowski, A. Pritchard, P. Rahkila, M. Rejmund, J. Saren, C. Scholey, S. Siem, M. G. Saint-Laurent, C. Simenel, Y. G. Sobolev, C. Stodel, J. Uusitalo, A. Villari, M. Bender, P. Bonche, and P. H. Heenen, Spectroscopy and single-particle structure of the odd-Z heavy elements ${ }^{255} \mathrm{Lr},{ }^{251} \mathrm{Md}$ and ${ }^{247} \mathrm{Es}$, Eur. Phys. J. A 30, 397 (2006)

[40] T. Nakatsukasa, K. Matsuyanagi, S. Mizutori, and Y. R. Shimizu, Microscopic structure of high-spin vibrational excitations in superdeformed ${ }^{190,192,194} \mathrm{Hg}$, Phys. Rev. C 53, 2213 (1996), arXiv:nucl-th/9512003

[41] K. Neergård and P. Vogel, On the microscopic description of nuclear vibrations when phonons occur at relatively low energy: (II). Octupole states of the even deformed nuclei with $A \geq 222$, Nucl. Phys. A 149, 217 (1970).

[42] R. V. Jolos, L. A. Malov, N. Y. Shirikova, and A. V. Sushkov, Structure of some low lying two-quasiparticle and collective states in nuclei with $Z \sim 100$ considered in the quasiparticle phonon model, J. Phys. G: Nucl. Part. Phys. 38, 115103 (2011).

[43] S. W. Yates, I. Ahmad, A. M. Friedman, K. Katori, C. Castaneda, and T. E. Ward, Configuration mixing of two-quasiparticle states in ${ }^{250} \mathrm{Cf}$, Phys. Rev. Lett. 36, 1125 (1976)

[44] K. Abusaleem, Nuclear Data Sheets for $A=249$, Nucl.
Data Sheets 112, 2129 (2011)

[45] National Nuclear Data Center, "Evaluated Nuclear Structure Data File", https://www.nndc.bnl.gov/ ensdf/

[46] H. Watanabe, G. Zhang, K. Yoshida, P. Walker, J. Liu, J. Wu, P. Regan, P.-A. Söderström, H. Kanaoka, Z. Korkulu, P. Lee, S. Nishimura, A. Yagi, D. Ahn, T. Alharbi, H. Baba, F. Browne, A. Bruce, R. Carroll, K. Chae, Z. Dombradi, P. Doornenbal, A. Estrade, N. Fukuda, C. Griffin, E. Ideguchi, N. Inabe, T. Isobe, S. Kanaya, I. Kojouharov, F. Kondev, T. Kubo, S. Kubono, N. Kurz, I. Kuti, S. Lalkovski, G. Lane, C. Lee, E. Lee, G. Lorusso, G. Lotay, C.-B. Moon, I. Nishizuka, C. Nita, A. Odahara, Z. Patel, V. Phong, Z. Podolyák, O. Roberts, H. Sakurai, H. Schaffner, C. Shand, Y. Shimizu, T. Sumikama, H. Suzuki, H. Takeda, S. Terashima, Z. Vajta, J. Valiente-Dóbon, and $\mathrm{Z}$. Xu, Long-lived $K$ isomer and enhanced $\gamma$ vibration in the neutron-rich nucleus ${ }^{172}$ Dy: Collectivity beyond double midshell, Phys. Lett. B 760, 641 (2016).

[47] G. Zhang, H. Watanabe, G. Dracoulis, F. Kondev, G. Lane, P. Regan, P.-A. Söderström, P. Walker, K. Yoshida, H. Kanaoka, Z. Korkulu, P. Lee, J. Liu, S. Nishimura, J. Wu, A. Yagi, D. Ahn, T. Alharbi, H. Baba, F. Browne, A. Bruce, M. Carpenter, R. Carroll, K. Chae, C. Chiara, Z. Dombradi, P. Doornenbal, A. Estrade, N. Fukuda, C. Griffin, E. Ideguchi, N. Inabe, T. Isobe, S. Kanaya, I. Kojouharov, T. Kubo, S. Kubono, N. Kurz, I. Kuti, S. Lalkovski, T. Lauritsen, C. Lee, E. Lee, C. Lister, G. Lorusso, G. Lotay, E. McCutchan, C.-B. Moon, I. Nishizuka, C. Nita, A. Odahara, Z. Patel, V. Phong, Z. Podolyák, O. Roberts, H. Sakurai, H. Schaffner, D. Seweryniak, C. Shand, Y. Shimizu, T. Sumikama, H. Suzuki, H. Takeda, S. Terashima, Z. Vajta, J. Valiente-Dóbon, Z. Xu, and S. Zhu, Interplay of quasiparticle and vibrational excitations: First observation of isomeric states in ${ }^{168}$ Dy and ${ }^{169}$ Dy, Phys. Lett. B 799, 135036 (2019)

[48] K. Yoshida and H. Watanabe, Enhanced collectivity of $\gamma$ vibration in neutron-rich Dy isotopes with $N=$ 108-110, Prog. Theor. Exp. Phys. 2016, 123D02 (2016), arXiv:1607.07111 [nucl-th]

[49] E. Browne and J. Tuli, Nuclear Data Sheets for $A=$ 251-259 (odd), Nucl. Data Sheets 114, 1041 (2013). 